\title{
Coupling of photoactive transition metal complexes to a functional polymer matrix ${ }^{* *}$
}

\author{
Miftahussurur Hamidi Putra, ${ }^{[\mathrm{a}]}$ Sebastian Seidenath, ${ }^{[\mathrm{b}]}$ Stephan Kupfer, ${ }^{[\mathrm{b}]}$ Stefanie $\mathrm{Gräfe}^{\left[{ }^{[b]}\right.}$ and \\ Axel Groß ${ }^{*[c]}$
}

\begin{abstract}
Conductive polymers represent a promising alternative to semiconducting oxide electrodes typically used in dye-sensitized cathodes as they more easily allow a tuning of the physicochemical properties. This can then also be very beneficial for using them in light-driven catalysis. In this computational study, we address the coupling of Ru-based photosensitizers to a polymer matrix by combining two different first-principles electronic structure approaches. We use a periodic density functional theory code to properly account for the delocalized nature of the electronic states in
\end{abstract}

the polymer. These ground state investigations are complemented by time-dependent density functional theory simulations to assess the Franck-Condon photophysics of the present photoactive hybrid material based on a molecular model system. Our results are consistent with recent experimental observations and allow to elucidate the light-driven redox chemical processes - eventually leading to charge separation - in the present functional hybrid systems with potential application as photocathode materials.

\section{Introduction}

The effective and efficient conversion of solar energy into electrical energy is challenging but might enable a significant reduction of the carbon emission in the world which will even have a beneficial economic impact. ${ }^{[1]}$ The market of solar cells is still dominated by silicon-based photovoltaic devices. ${ }^{[2-4]}$ However, dye-sensitized solar cells (DSSC) ${ }^{[5]}$ have the potential to become an alternative to silicon-based solar cells, as they are associated with a very low weight, low costs, ease of

[a] M. H. Putra

Institute of Theoretical Chemistry,

Ulm University

89069 Ulm, Germany

[b] S. Seidenath, Dr. S. Kupfer, Prof. Dr. S. Gräfe

Institute for Physical Chemistry (IPC) and Abbe Center of Photonics,

Friedrich Schiller University Jena,

Helmholtzweg 4, 07743 Jena

[c] Prof. Dr. A. Groß

Institute of Theoretical Chemistry,

Ulm University,

89069 Ulm, Germany,

and

Helmholtz Institute UIm (HIU)

Electrochemical Energy Storage,

89069 Ulm, Germany

E-mail: axel.gross@uni-ulm.de

${ }^{[*}$ ] A previous version of this manuscript has been deposited on a preprint server (https://doi.org/10.33774/chemrxiv-2021-2wIII).

$\square$ Supporting information for this article is available on the WWW under https://doi.org/10.1002/chem.202102776

in Part of a Special Issue on Contemporary Challenges in Catalysis.

of $(\odot) 2021$ The Authors. Chemistry - A European Journal published by WileyVCH GmbH. This is an open access article under the terms of the Creative Commons Attribution Non-Commercial License, which permits use, distribution and reproduction in any medium, provided the original work is properly cited and is not used for commercial purposes. production, and can be used in a more flexible manner than the conventional silicon-based technology. ${ }^{[6-10]}$

In general, there are two kinds of DSSCs, i.e. $n$-type and $p$ type. In $n$-type DSSCs, the electrons are injected from the dye to the photoanode (mainly $\mathrm{TiO}_{2}$ ). In contrast, hole injection occurs in $p$-type DSSCs from the dyes to the photocathode made of, e.g., NiO. ${ }^{[1-13]}$ Typically, $p$-type DSSCs feature an efficiency of about $2.5 \%$, roughly one order of magnitude lower than the efficiency of $n$-type DSSCs (13.1\%). ${ }^{[14]}$ Interestingly enough, tandem systems combining $n$-type with $p$-type DSSCs were predicted to achieve efficiencies of up to around $40 \% .{ }^{[14]}$ Still, there is a strong need to increase the efficiency of $p$-type DSSCs to make them more competitive, e.g. by developing new photocathode materials with adjustable electronic properties. In this sense, replacing inorganic by organic cathode materials is highly promising.

The fundamental concept as well as the underlying lightdriven processes in $p$-type DSSCs are illustrated in Figure 1. Firstly, an electron of the dye is excited by a photon into an excited state. Subsequently, the hole is injected from the dye to the highest occupied molecular orbital (HOMO) of the photocathode and the hot electron is transferred from the dye to the redox or catalytic agent. ${ }^{[13,14]}$ In the operation of the DSSC, undesired electron transfer pathways should be suppressed. Such undesired processes are, for example, the electron transfer from the dye to the photocathode or the direct recombination of the hole and the electron from the excited state of dye to the HOMO of the photocathode. ${ }^{[14]}$ In addition, the electron may also be transferred from the excited state of the photocathode to the dye, as the energy gap of this semiconductor is typically comparable to the energy gap of the dye. ${ }^{[15]}$

The concept of $p$-DSSCs can also be extended to photochemical molecular devices (PMD) by combining a photosensitizer and a catalyst connected by an electron relay. ${ }^{[16-18]}$ In 


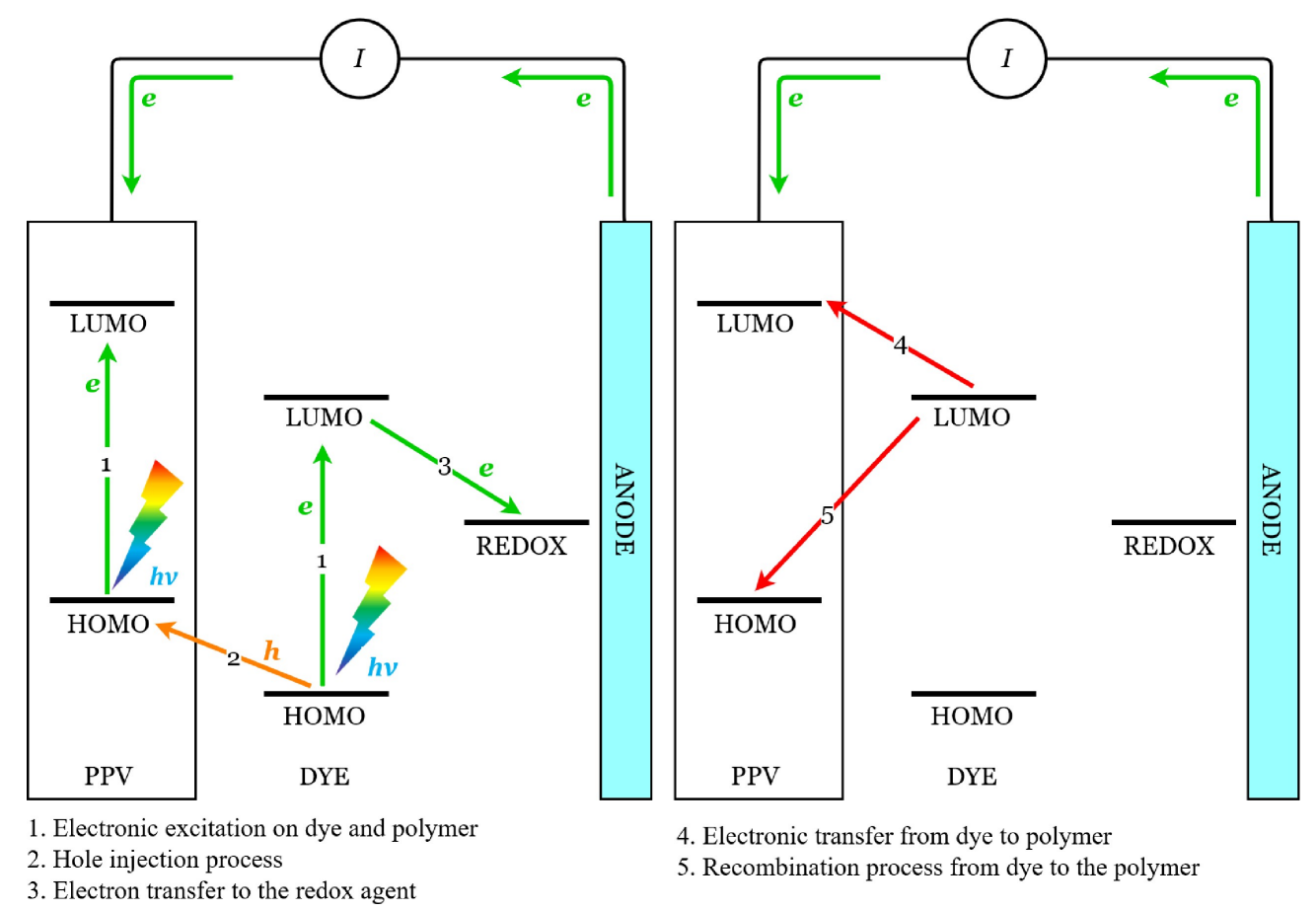

Figure 1. Schematic presentation of the processes occurring in a $p$-DSSC of a photoactive complex coupled to a PPV polymer including the full DSSC circuit. The left panel illustrates the beneficial processes whereas the right panel depicts the parasitic phenomena.

such a PMD, the electron is directly transferred to the catalytic agent, e.g. a hydrogen evolution catalyst, instead to a redox agent, such as $\mathrm{I}_{3}^{-} / \mathrm{I}^{-[11,13,16,18]}$ Furthermore, apart from just addressing the properties of the DSSCs, hole conducting organic polymers have been put forward as viable alternatives to $\mathrm{NiO}$ or other metal oxide materials, as the tuning of the physicochemical properties of polymers is easier ${ }^{[19]}$ than those of $\mathrm{NiO}$.

Thus the integration of molecular light-driven catalysts into soft matter matrices presents an attractive route for tailored photochemical molecular devices as the embedding can give control over charge transfer processes, photochemical reactivity and degradation resistance. ${ }^{[20]}$ However, in order to do this in a rational fashion, it is necessary to understand the properties of polymers and its coupling to the photosensitizer. In order to contribute to an improved understanding of this coupling, we have performed quantum chemical calculations on a soft matter embedded $\mathrm{Ru}$ (II)-based photosensitizer. The choice of the considered systems has been motivated by a recent experimental study. ${ }^{[15]}$ In order to properly capture both the delocalized nature of electronic states of the polymers as well as the local nature of the electronic excitations of the photoactive complex, we combined two complementary approaches: On the one hand, we have carried out periodic density functional theory (DFT) calculations modelling in principle an infinitely long polymer coupled to a periodic array of photoactive transition metal complexes. This allows to capture the delocalized nature of the electronic system of the polymers. On the other hand, in order to assess light-driven processes such as electronic excitation spectra, we employed time-dependent density functional theory (TDDFT) onto local models of the complexes.

The organization of this paper is as follows: We will first present details of the quantum chemistry methods used in this work. Then, the results of our quantum chemistry calculations will be presented and discussed together with a comparison with the available experimental data. Finally, conclusions are provided.

\section{Computational Details}

In the first computational approach used in the study, we modeled the polymer as an infinite chain with a periodic set-up. ${ }^{[21]}$ As the polymer, we considered the conductive polymer poly(p-phenylenevinylene) (PPV) as functionalized PPV derivates are commonly used in polymer solar cells $s^{[22]}$ and have already been functionalized with a Ru(II)-polypyridyl-based photosensitizer. ${ }^{[15]}$ In this experiment, ${ }^{[15]}$ two photosensitizers with either an electron-withdrawing ethyl ester group (-COOEt) or an electron-donating tertbutyl group as substituent on their bipyridine ligands were considered. In order to reduce the numerical effort, in the calculations we replaced these substituents by smaller electronwithdrawing and -donating groups, $-\mathrm{COOH}$ (Ru1) and $\mathrm{CH}_{3}$ (Ru2), respectively, which, however, mimic the electronic effects of the substituents used in the experiment. The structure of the polymer is illustrated in Figure 2a, while the PPV-embedded photosensitizers (Ru1 and Ru2) - chemically linked by alkyl side chains - are depicted in Figure 2b. In the periodic cell, we include four repeat units of the polymer and replace the remaining $\mathrm{C}_{7} \mathrm{H}_{15}$ alkyl chains by a methyl group to reduce the computational effort. Such a substitution is known to only slightly modify the electronic structure of the polymer. ${ }^{[23]}$ Furthermore, we also included two $\mathrm{PF}_{6}^{-}$ 
anions in the periodic unit cell, as indicated in Figure $2 \mathrm{~b}$, in order to counter-balance the charge $(+2)$ of the ruthenium complex.

The periodic quantum chemical calculations have been performed employing density functional theory (DFT) as implemented in the FHI-AIMS $\operatorname{code}^{[24,25]}$ that utilizes a basis of numeric atom-centered orbitals. This allows an efficient parallelization of the periodic DFT calculations, even for hybrid functional calculations ${ }^{[26-28]}$ which are typically very time-consuming for infinite systems. To perform the geometry optimization, we used the Perdew-Burke-Ernzerhof (PBE) exchange-correlation functional within the generalized gradient approximation (GGA) ${ }^{[29]}$ with a light basis set. ${ }^{[24]}$ The van der Waals dispersion interaction is taken into account using the TkatchenkoScheffler approach. ${ }^{[30]}$ Relativistic energy corrections are included within the ZORA approximation. ${ }^{[31]}$ The PBE functional like all GGA functionals underestimates the energy gap. In order to obtain a reliable electronic structure of the polymer linked to the $\mathrm{Ru}(\mathrm{II})$ dye, we performed single points calculations of the relaxed structure obtained from PBE optimization by means of hybrid functionals such as B3LYP and PBE0. ${ }^{[32-34]}$

To describe the PPV polymer alone, we used a cell size $6.8 \times 40 \times 25 \AA^{3}$ with a $8 \times 1 \times 1 k$-point set for the geometry optimization and a $16 \times 1 \times 1 \mathrm{k}$-point set for the determination of the electronic gap. For the combined PPV $+\mathrm{Ru}$ system, we extended the unit cell to a $(4 \times 1 \times 1)$ supercell with respect to the polymer repeat units with a cell size of $27.2 \times 40 \times 40 \AA^{3}$. Because of the significantly larger unit cell, we used a $1 \times 1 \times 1 k$-point set for the PPV + Ru system for both the geometry optimization as well as for the determination of the electronic structure.

In order to estimate the change of the properties of polymer and the dye, we determined the electronic properties of short PPV oligomers, i.e. the monomer, dimer and trimer, and the photo- sensitizer in various implicit solvents, such as gas phase, toluene, acetone, acetonitrile and water represented through their corresponding dielectric constants. These calculations were performed with the Gaussian 16 package. ${ }^{[35]}$ We used PBEO as the exchangecorrelation functional $^{[32]}$ and the D3 dispersion correction scheme with BJ damping to account for dispersion effects. ${ }^{[36]}$ The implicit solvent was model employing the SMD method. ${ }^{[37]}$

In the analysis of our results, we use the concept of the hole injection efficiency (HJE) $\Delta E_{\mathrm{HJE}}{ }^{[11,38-40]}$ which corresponds to the difference in the energy levels of the HOMO at the dye and the polymer,

$$
\Delta E_{\mathrm{HJE}}=E_{\mathrm{Dye}}^{\mathrm{HOMO}}-E_{\mathrm{PPV}}^{\mathrm{HOMO}},
$$

and which allows to estimate the charge transfer between polymer and dye. On the other hand, a large negative hole injection energy $\Delta E_{\mathrm{HJE}}$ corresponds to an indication that hole injection might in fact. be more favorable which can be quantified using the electron injection energy $\Delta E_{\mathrm{EJE}}$, which is given by the difference in the energies of the lowest unoccupied molecular orbital (LUMO),

$$
\Delta E_{\mathrm{EJE}}=E_{\mathrm{PPV}}^{\mathrm{LUMO}}-E_{\mathrm{Dye}}^{\mathrm{LUMO}} .
$$

Equivalently, a large negative value of $\Delta E_{\mathrm{EJE}}$ indicates a higher likelihood for an electron transfer process from the dye to the polymer. Consequently, efficient $p$-DSSCs are characterized by a small negative or even positive value of $\Delta E_{\mathrm{EJE}}$ and a large negative value of $\Delta E_{\mathrm{HJE}}$

In order to directly access the excited states involved in a charge separation process, we also employed Kohn-Sham time-dependent

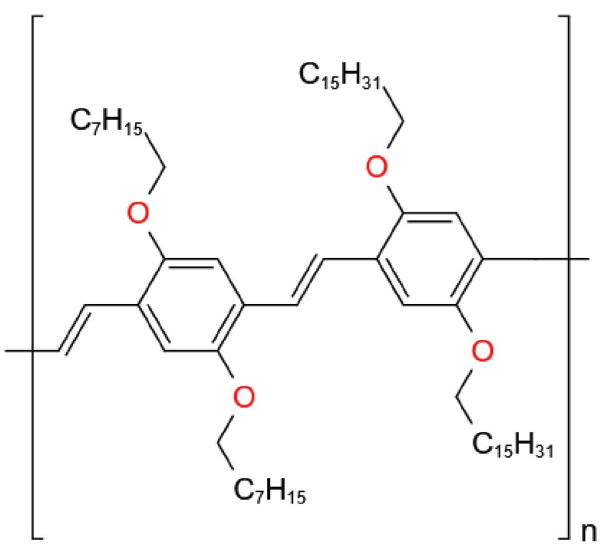

(a)

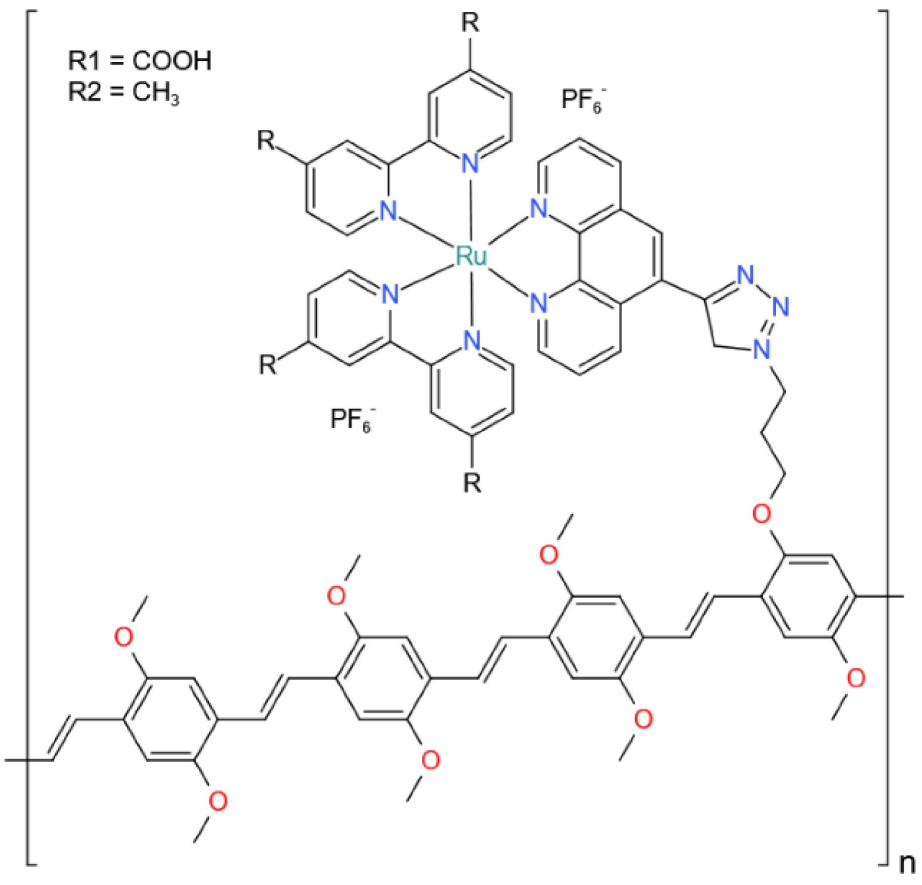

(b)

Figure 2. (a) Structure of PPV polymer; alkyl chains are replaced by methyl groups to reduce the computational cost. (b) Unit cell used in the periodic DFT calculations to model the polymer and the Ru(II) dye linked to the polymer. Structure of the Ru(II)-based photosensitizer linked to the PPV polymer (PPV-Ru) together with two $\mathrm{PF}_{6}{ }^{-}$counter ions for charge balancing. Two different substituents have been used, $\mathrm{R}=\mathrm{COOH}$ and $\mathrm{R}=\mathrm{CH} \mathrm{H}_{3}$, yielding the hybrid materials PPV-Ru1 and PPV-Ru2, respectively. 
density functional theory (TDDFT). Thus, excitation energies, oscillator strengths, transition dipoles and excited state densities are available for analysis. A major advantage compared to simpler models is the intrinsic treatment of dynamic electron correlation in DFT - an advantage that excited state calculations using TDDFT inherit. In particular, TDDFT struggles with describing Rydberg or charge transfer states, which can be overcome by applying rangeseparated or hybrid functional with a high degree of exact HF exchange. ${ }^{[41-43]}$ In this case, the PBE0 functional (25\% HF exchange) was chosen as previous joint spectroscopic-theoretical studies highlighted its capability to unambiguously assess the FranckCondon photophysics, i.e. excitation energy, transition dipole moments, electronic characters and excited state gradients, of transition metal complexes. ${ }^{[32,44,45]}$

Still, periodic DFT codes typically do not contain TDDFT implementations to derive oscillator strengths. Therefore, we used local models of the extended PPV-Ru systems based on the periodic structures obtained in the calculations described above in order to account for the computational demands of TDDFT calculations. We took the frozen sequence of four benzene units with one Ru dye from the unit cell used in the periodic DFT calculations depicted in Figure $2 \mathrm{~b}$ as input for our TDDFT calculations. The $\mathrm{C}-\mathrm{C}$ bonds broken in this process were capped by hydrogen atoms. The positions of these hydrogens were then optimized at the def2-SVP/ $\mathrm{PBE}^{[29,46]}$ level of DFT using the ORCA program suite ${ }^{[47,48]}$ while the remaining atoms remained frozen.

TDDFT calculations were done using the NWChem code ${ }^{[49]}$ with the PBEO functional to maintain comparability with all other results. The def2-SVP basis set was used together with its corresponding ECPs while the Tamm-Dancoff approximation (TDA) was applied. ${ }^{[46,50,51]}$ All calculations were done in the gas phase. The lowest 100 singlet states were calculated, covering an energy range of up to $4.1 \mathrm{eV}$ $(302 \mathrm{~nm})$.

\section{Results and Discussion}

In order to validate the reliability of our results, we start the presentation of our results with a comparison of the calculated electronic properties of the PPV polymer, such as the work function, the position of HOMO and LUMO levels, and the energy gap, with the corresponding experimental results ${ }^{[15,52-55]}$ (see Table 1). We have evaluated the electronic properties of the polymer in the structure relaxed with the PBE functional using the different functionals, $\mathrm{PBE}$ and the hybrid functionals B3LYP and PBEO. The position of HOMO and LUMO levels are given with respect to the vacuum level. First of all the very wellknown fact is reproduced that GGA functionals such as PBE severely underestimate HOMO-LUMO gaps ${ }^{[21,56]}$ which can be traced back to unphysical self-Coulomb repulsion contained in these functionals. ${ }^{[57,58]}$ In contrast, the hybrid functionals, i.e. PBEO and B3LYP, including a certain portion of exact exchange do a much better job in this respect.

Among the two hybrid functionals, PBEO outperforms B3LYP with respect to the position of the HOMO and LUMO levels and the energy gap, as far as the comparison with the experiment is concerned. Interestingly enough, PBE reproduces the measured position of the LUMO level better than the two hybrid functionals with PBEO exhibiting the largest deviation with respect to the experiment. Still we will use PBEO in the following in the discussion of the electronic properties of the PPV-Ru systems as it yields the best agreement with the measured electronic levels of the PPV polymer.

Now, we consider the electronic levels of the joint system with the Ru dye linked to the PPV polymer. As a first step, we show in Figure 3 the band structures of the polymer alone and the two joint systems calculated with the PBEO functional. In addition, the total density of states (DOS) and the local density of states (LDOS) projected onto the polymer, the Ru atom, and the phenatroline and bipyridine moiety of the photosensitizer are plotted. Figure $3 a$ illustrates the one-dimensional band structure of the isolated PPV polymer. The polymer has a direct band gap at the $\Gamma$ point which widens towards the $X$ point. Note that the variation of the electronic bands with the wavevector $k$, the dispersion, is a measure of the delocalization, localized states just have a flat dispersion. The changes of the bandstructure upon attaching the Ru complex with the $-\mathrm{COOH}$ substituent to the polymer (PPV-Ru1) are shown in Figure 3b. In addition to the PPV states, now a high number of dispersionless, i.e., $k$-independent states appears. The vanishing dispersion indicates that these are localized states of the Ru complex. In fact, the lowest unoccupied state is also dispersionless and located at the phenatroline and bipyridine moiety of the photosensitizer, as the local density of states plotted in Figures $3 \mathrm{c}$ and $\mathrm{e}$ indicates. This demonstrates that the LUMO of the combined system is located within the photoactive complex. Furthermore, the plotted DOS also confirms that the HOMO remains to be located at the polymer. The PPV bands hardly change upon the attachment of the Ru complex showing that there is obviously a rather small coupling between the electronic states of the PPV and the Ru complex.

Upon changing the substituent to $-\mathrm{CH}_{3}$ (PPV-Ru2), the PPVderived band structure hardly changes, as Figure $3 \mathrm{~d}$ shows, but the positions of the dispersionless states are altered. This becomes even more obvious upon comparing the densities of state in Figure 3e and Figure 3c. The peaks of the LDOS at the $\mathrm{Ru}$ atom are at different energetic positions. Furthermore, the

\begin{tabular}{|c|c|c|c|c|c|}
\hline No. & $\begin{array}{l}\text { XC } \\
\text { Corr. }\end{array}$ & $\begin{array}{l}\text { Energy Gap } \\
\text { (eV) }\end{array}$ & $\begin{array}{l}\Phi \\
(\mathrm{eV})\end{array}$ & $\begin{array}{l}\text { HOMO } \\
(\mathrm{eV})\end{array}$ & $\begin{array}{l}\text { LUMO } \\
(\mathrm{eV})\end{array}$ \\
\hline 1 & PBE & 1.0144 & 3.521 & -4.052 & -3.038 \\
\hline 2 & B3LYP & 2.017 & 3.210 & -4.650 & -2.633 \\
\hline 3 & PBEO & 2.271 & 3.102 & -4.824 & -2.554 \\
\hline 4 & Exp. & $2.1-2.4^{[15,52-54]}$ & $4.000^{[55]}$ & -4.90 to $-5.41^{[15,52-54]}$ & -2.70 to $-3.01^{[15,52-54]}$ \\
\hline
\end{tabular}



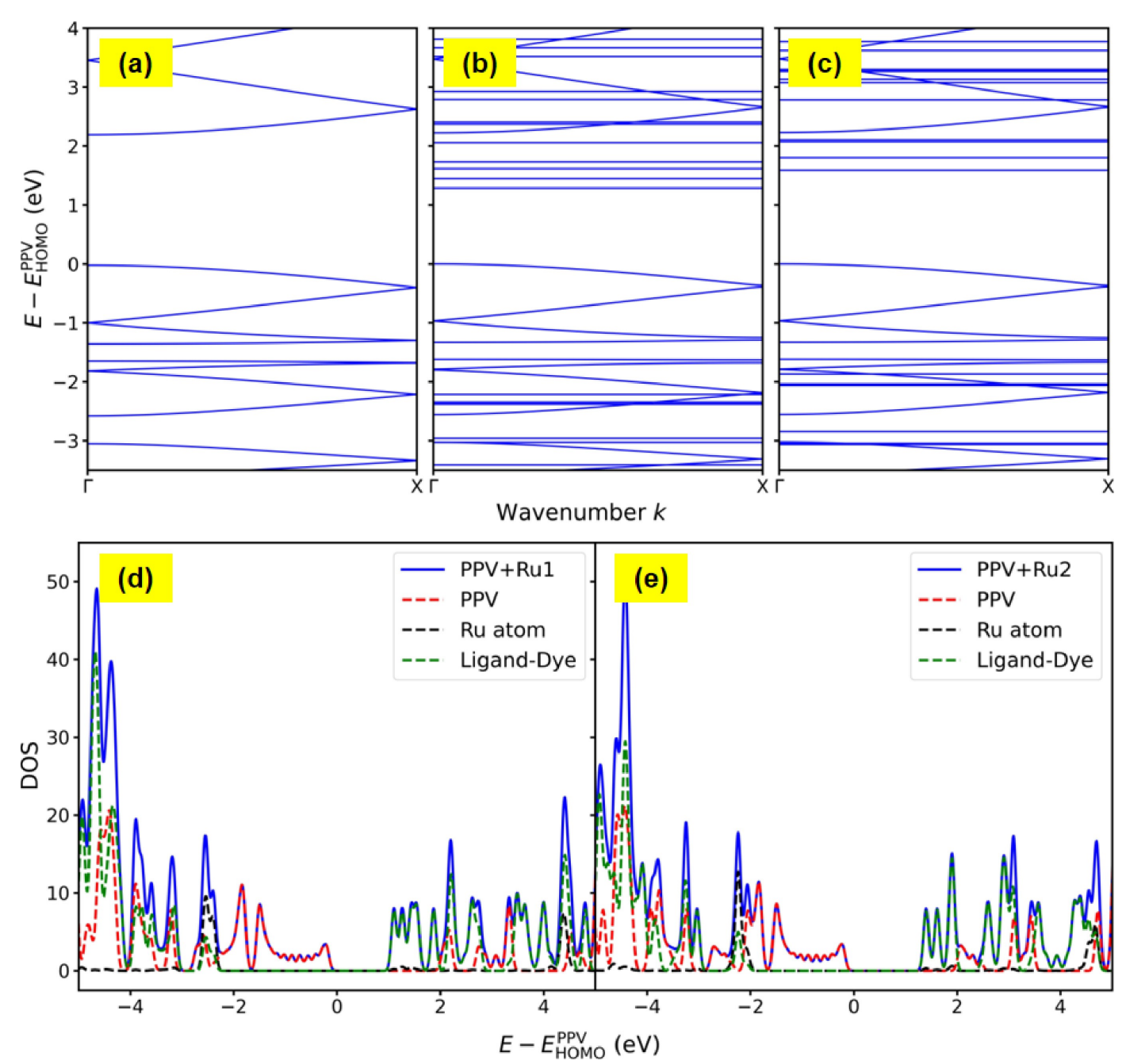

Figure 3. Bandstructures and densities of states of the PPV polymer alone and coupled to the Ru photosensitizer with - $\mathrm{COOH}$ (PPV-Ru1) and - $\mathrm{CH}$ ( $(\mathrm{PPV}-\mathrm{Ru} 2)$ substituents in the gas phase calculated with the PBE0 functional. a, b, d) Band structure of the isolated PPV polymer, the PPV-Ru1 and the PPV-Ru2 systems, respectively; c, e) density of the states (DOS) of the PPV-Ru1 and the PPV-Ru2 systems, respectively. In addition to the total DOS, we also plot the local DOS of the PPV polymer, of the Ru center and of the phenatroline and bipyridine moiety denoted by Ligand-Dye. In the presentation of the DOS, a smearing of $50 \mathrm{meV}$ has been applied.

LUMO of the Ru2 complex is found at higher energies with respect to the valence band edge of the polymer, overall a considerably modified electronic structure results. In summary, the results presented in Figure 3 show that the electronic properties of the polymer are hardly modified by attaching the Ru complex, irrespective of the nature of the two substituents used. The electronic states of the Ru complex hardly couple to the electronic states of the polymer, but they are sensitive to the nature of the substituent being used.

Next, we turn to a local picture of the electronic orbitals which has been obtained by extracting the position-resolved variation of the HOMO and the LUMO, as illustrated in Figure 4. There, also the corresponding energy levels of the PPV-Ru with the $-\mathrm{COOH}$ and $-\mathrm{CH}_{3}$ substituents are given, now with respect to the vacuum level in order to account for work function effects. In fact, our analysis of the spatial distribution of the electronic orbitals of the $\mathrm{Ru}$ photosensitizer linked to the polymer only yields orbitals that are almost entirely located either on the polymer or on the photosensitizer so that the distinction between polymer and photosensitizer orbitals is unique which confirms all the findings we derived from the analysis of the band structure and the density of states. Obviously, the polymer and the attached photosensitizer are not strongly electronically coupled. Thus, the alkyl chain connector considered here apparently acts as a geometric rope immobilizing the photosensitizer rather than as an electronic wire coupling the electronic states of polymer and the photosensitizer.

As Figure 4 confirms, the energies of the HOMO and the LUMO localized on the polymer with the two different substituents are very similar, and the HOMO-LUMO gap is almost identical. Hence, this again shows that the substituents do apparently not have a significant influence on the electronic properties of the polymer which is not too surprising considering the fact that they are not directly interacting with the polymer. Furthermore, a closer analysis yields that the HOMO 


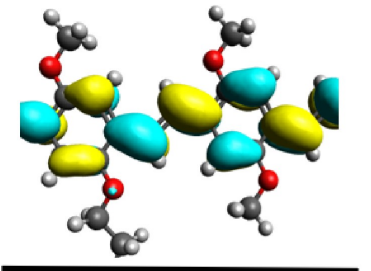

$$
E=-2.77 \mathrm{eV}
$$

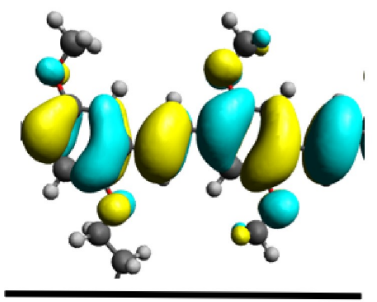

$E=-5.04 \mathrm{eV}$
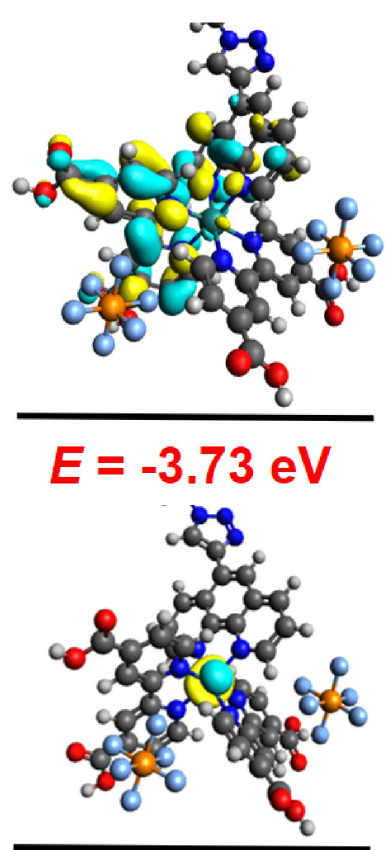

$E=-7.22 \mathrm{eV}$

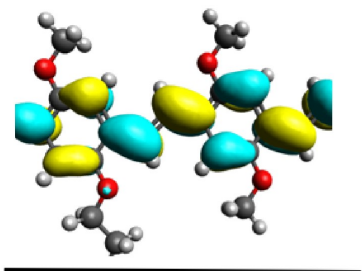

$E=-2.81 \mathrm{eV}$

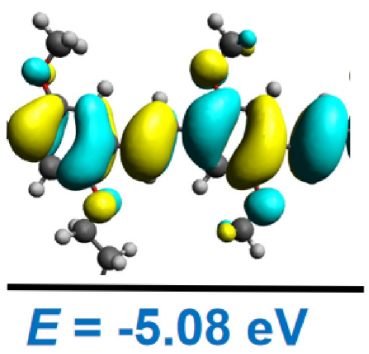

8
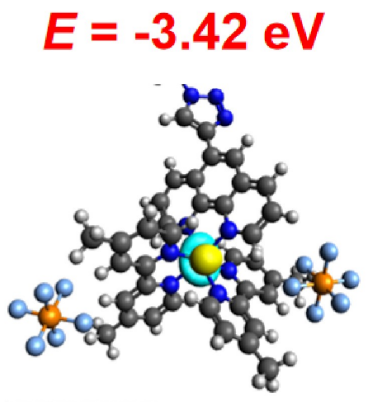

$E=-6.88 \mathrm{eV}$

(a)

(b)

Figure 4. HOMO and the LUMO levels of the PPV polymer and the Ru photosensitizer with the $-\mathrm{COOH}$ (a) and $-\mathrm{CH}_{3}$ (b) substituents, respectively, in the gas phase taken with respect to the electronic vacuum level. The numbers in blue represent the HOMO-LUMO levels of PPV, those in red color denote the HOMOLUMO levels of the photosensitizer. These levels have been identified through a charge analysis of the electronic orbitals which is illustrated by the charge isosurfaces in the ball-and-stick presentation of the polymer and the Ru photosensitizer.

on the polymer has a $\pi$-orbital character whereas the LUMO is of $\pi^{*}$-orbital character. Interestingly, the HOMO and LUMO levels localized on the Ru photosensitizer differ by a larger amount, as indicated by the different substitution pattern with the electron-withdrawing $(-\mathrm{COOH})$ vs. the electron-donating $\left(-\mathrm{CH}_{3}\right)$ substituents. The corresponding levels of the Ru1 complex with the $-\mathrm{COOH}$ substituent are by about $0.3 \mathrm{eV}$ lower than the Ru2 complex with the $-\mathrm{CH}_{3}$ substituent, also in agreement with the results shown in Figure 3. As Figure 4 illustrates, for both substituents the HOMO of the photosensitizer remains to be localized within the $d$-orbitals at the ruthenium metal center. On the other hand, the spatial distribution of the LUMO depends on the choice of the substituent. For the $-\mathrm{COOH}$ substituent, the electron within the LUMO are accumulated at the $\pi^{*}$-orbital of the bipyridine moiety, whereas in the $-\mathrm{COOH}$ case the LUMO electrons are concentrated in the $\pi^{*}$-orbital of the phenantroline moiety.

The calculated hole and electron injection energies $\Delta E_{\mathrm{HJE}}$ and $\Delta E_{\mathrm{EJE}}$ for the polymer and the photosensitizer in the gas phase have been listed in Table 2 in order to qualitatively assess the hole and electron transfer. The hole injection energy $\Delta E_{\mathrm{HJE}}$ with respect to the PPV-Ru1 combination is by about $0.4 \mathrm{eV}$ lower than the one of the PPV-Ru2 combination. Hence we predict that the PPV-Ru1 system is associated with a more

Table 2. Position of the HOMO and the LUMO and hole and electron injection efficiencies $\triangle E_{\mathrm{HJE}}$ and $\triangle E_{\mathrm{EJE}}$ calculated for the PPV-Ru system in gas phase and with $\mathrm{N}_{\mathrm{H}_{2} \mathrm{O}}$ water molecules explicitly included. All experimental values are derived from Ref. [15]. The experimental HOMO and LUMO level are estimated by the corresponding redox levels, and the hole and electron injection efficiencies are derived from the measured cyclic voltammograms. All values are given in eV.

\begin{tabular}{|c|c|c|c|c|c|c|c|c|}
\hline Parameter & $\begin{array}{l}\text { Gas Phase } \\
\text { Ru1 }\end{array}$ & Ru2 & $\begin{array}{l}N_{\mathrm{H}_{2} \mathrm{O}}=10 \\
\text { Ru1 }\end{array}$ & Ru2 & $\begin{array}{l}\mathrm{N}_{\mathrm{H}_{2} \mathrm{O}}=30 \\
\text { Ru1 }\end{array}$ & Ru2 & $\begin{array}{l}\text { Experi } \\
\text { Ru1 }\end{array}$ & Ru2 \\
\hline$E_{\mathrm{PPV}}^{\mathrm{HOMO}}$ & $-5.040-$ & -5.083 & -4.999 & -5.012 & -4.921 & -4.987 & -5.41 & -5.41 \\
\hline$E_{\mathrm{PPV}}^{\mathrm{HOMO}}$ & -2.774 & -2.812 & -2.729 & -2.740 & -2.681 & -2.709 & -3.01 & -3.01 \\
\hline$E_{\text {Dye }}^{\text {LUMO }}$ & -7.222 & -6.884 & -6.932 & -6.612 & -6.687 & -6.372 & -5.78 & -5.50 \\
\hline$E_{\text {Dye }}^{\text {LUMO }}$ & -3.729 & -3.415 & -3.487 & -2.968 & -3.162 & -2.713 & -3.35 & -3.03 \\
\hline$\Delta E_{\mathrm{HJE}}$ & -2.182 & -1.801 & -1.933 & -1.601 & -1.766 & -1.386 & -0.37 & -0.09 \\
\hline$\Delta E_{\mathrm{EJE}}$ & 0.955 & 0.604 & 0.758 & 0.228 & 0.482 & 0.004 & 0.34 & 0.02 \\
\hline
\end{tabular}


efficient hole transfer than PPV-Ru2. On the other hand, PPV-Ru2 has a electron injection energy $\Delta E_{\mathrm{EJE}}$ that is lower by about $0.35 \mathrm{eV}$ than $\Delta E_{\mathrm{EJE}}$ of PPV-Ru1. This indicates that PPV-Ru2 will have a faster charge recombination than PPV-Ru1.

These findings are confirmed by the transient absorption spectroscopy measurement ${ }^{[15]}$ which find that the hole injection process on PPV-Ru1 occurs within $49 \pm 4$ ps which is significantly shorter that the hole injection time $86 \pm 9$ ps for PPV-Ru2. Furthermore, PPV-Ru1 also has a longer lifetime of the charge separated state $(1587 \pm 201 \mathrm{fs})$ than PPV-Ru2 $\left(994 \pm 203\right.$ ps). ${ }^{[15]}$ This comparison with the experiment indicates that the analysis of $\Delta E_{\mathrm{HJE}}$ and $\Delta E_{\mathrm{EJE}}$ indeed allows to qualitatively predict the hole injection and charge recombination properties of a conjugated polymer functionalized with $\mathrm{Ru}$ photosensitizers.

Still, such a photocathode system is usually not operated under gas-phase conditions but in the presence of an electrolyte. ${ }^{[15]}$ In fact, it is well-known that the presence of an electrolyte can significantly modify the electronic properties of photoactive complexes. ${ }^{[59,60]}$ In a first step, we calculated the electronic properties of the isolated PPV oligomers (monomer, dimer, and trimer) and the Ru2 dye separately in the presence of implicit solvents. In the case of the dye, we added two $\mathrm{PF}_{6}^{-}$ counter ions in order balance the positive charge of the Ru dye. The HOMO, LUMO and the energy gap of the considered systems are plotted as a function of the dielectric constant of the solvent in Figure 5. In general, we find a relatively weak dependence of these electronic properties of the PPV oligomers on the dielectric constant. There is a slight downshift of the energy levels of the HOMO and the LUMO with increasing dielectric constant, but the absolute shifts are below $0.25 \mathrm{eV}$. On the other hand, introducing implicit solvents modifies the HOMO and LUMO levels of the Ru2 dye complex significantly. Both the HOMO and the LUMO are shifted up by up to $1 \mathrm{eV}$ with increasing dielectric constant, but in such a way that the HOMO-LUMO gap after an initial sharp rise then decreases again. Thus the electronic levels of the dye are obviously more sensitive with respect to the presence of a solvent than the electronic levels of the polymer.

As the FHI-AIMS code used in this study does not allow to include implicit solvents in its implementation for periodic systems, we tried to estimate the effect of the presence of a solvent by adding $\mathrm{N}_{\mathrm{H}_{2} \mathrm{O}}=10$ and 30 explicit water molecules to the simulation cell and relaxing them. Note that by this procedure the statistical nature of liquid water is not properly accounted for, however, performing a statistical average over many possible water structures would be much too timeconsuming. The calculated energy levels and the hole and electron injection efficiences without and with water molecules are presented in Table 2. We also compare the calculated values with the experiment. ${ }^{[15]} \Delta E_{\mathrm{HJE}}$ and $\Delta E_{\mathrm{EJE}}$ are estimated from the experiment by analyzing the cyclic voltammograms in Ref. [15], and the HOMO and LUMO levels are estimated from the reported oxidation and reduction levels, as done before. ${ }^{[61-63]}$

According to Table 2, the addition of the water molecules hardly changes the local HOMO and LUMO levels on the PPV polymer which is in nice agreement with the calculations including implicit solvents presented in Figure 5a. However, the HOMO and LUMO levels are shifted up by about $0.6 \mathrm{eV}$ upon adding 30 water molecules to the simulation cell, again in good agreement with the implicit solvent results (Figure $5 b$ ). Consequently, also the values of the hole and electron injection efficiency $\Delta E_{\mathrm{HJE}}$ and $\Delta E_{\mathrm{EJE}}$ become modified, both are reduced by about $0.4 \mathrm{eV}$ upon adding 30 explicit water molecules.

As far as the comparison between calculated and measured values listed in Table 2 is concerned, a qualitative and almost semi-quantitative agreement is observed. This is rather satisfying given first the uncertainties of the derivation of the numbers from the experimental results. Second, the regular periodic structure of the PPV-Ru system illustrated in Figure $2 b$ certainly represents an idealization with respect to the periodicity of the attached photosensitizer, the assumption of a straight polymer, the exact length of the linker between polymer and photosensitizer and the representation of the aqueous environment by a finite number of explicit water

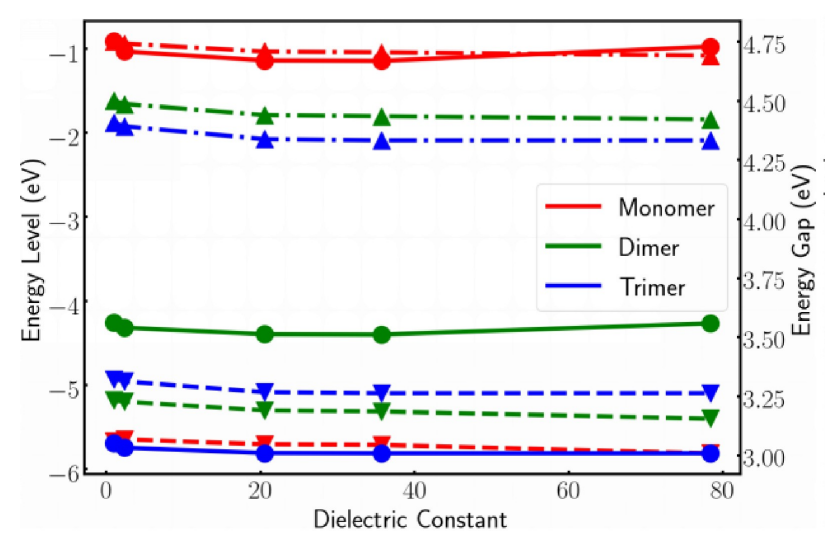

(a)

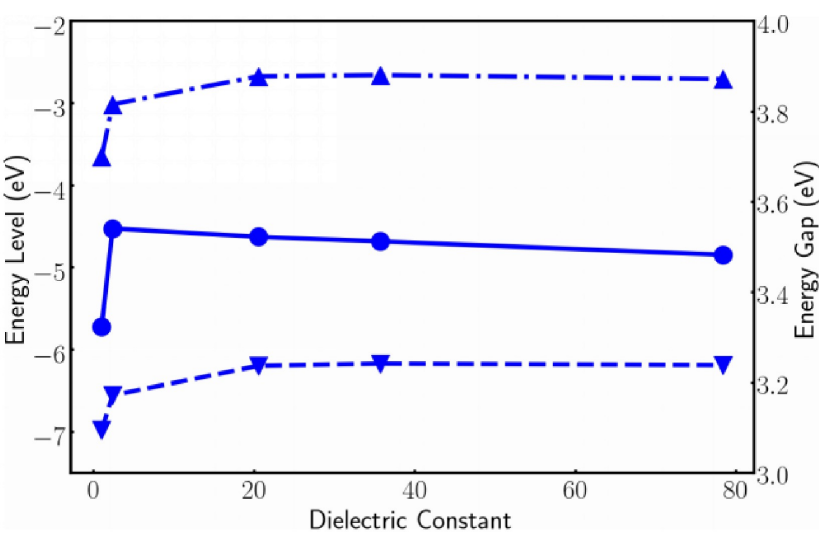

(b) 
molecules in just one structure. Still, our DFT calculations yield a HOMO-LUMO gap of the Ru photosensitizer that is by roughly $1 \mathrm{eV}$ larger than the experimentally derived value which has also been found in similar systems. ${ }^{[64,65]}$

Time-dependent density functional theory, on the other hand, is well established to access and predict the light-driven processes in transition metal complexes ${ }^{[44,66]}$ and soft matter embedded light-harvesting units. ${ }^{[67]}$ Through TDDFT, individual excited states and their properties such as oscillator strength and electron density become accessible, which allows fundamental insights into the mechanisms of light-driven processes such as electron or hole injection. Further, orbital-based KohnSham TDDFT has the advantage of providing easy means of visualization in the familiar orbital picture or with charge density difference plots. It is an excellent method to couple with the experiment: Actual measurements help verify calcu- lated data, while the calculation can access properties that are difficult to ascertain for the experimentalist, such as the presence of optically inaccessible "dark" states, such as the desired charge-transfer states in the present hybrid material. Therefore, as a complementary approach, we address the PPV-Ru complexes also by orbital-based TDDFT calculations using local models for the complexes.

A first comparison between TDDFT calculations and experiment is given by the comparison of the TDDFT spectra with experimentally measured ones. Figure 6 shows calculated and experimental spectra for PPV-Ru1 and PPV-Ru2 side by side. The spectra are in good agreement, especially considering the simplified geometries, omitted solvent and computational concessions towards the basis size. Table 3 lists the most prominent excitations in detail, while their electronic nature is
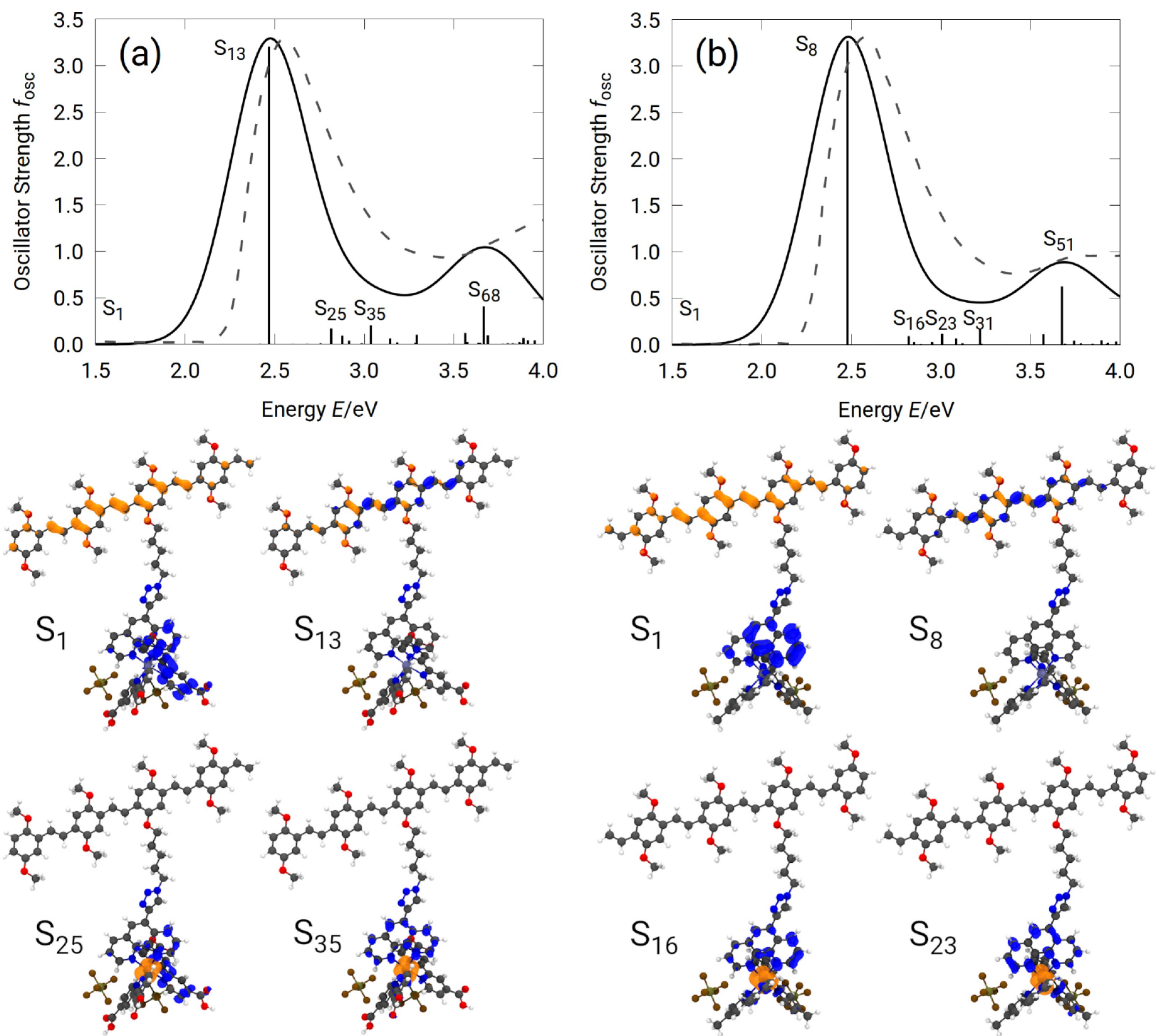

Figure 6. Visualization of calculated UV/Vis absorption spectra (solid) and excitations for PPV-Ru1 (a) and PPV-Ru2 (b). Experimental spectra (dashed) are adapted from Ref. [15] and have been scaled arbitrarily. Calculated transitions have been broadened to a full width at half maximum of 0.5 eV. Charge density difference plots (isovalue $=0.002$ ) for some important transitions are included. Holes are depicted in orange, electrons in blue. Calculated data was obtained at the PBE0/def2-SVP level of TDDFT. Energies and oscillator strengths can be found in Table 3 . 
Table 3. Selected excitations contributing to UV/Vis absorption spectra of PPV-Ru1 and PPV-Ru2 as well as (dipole-forbidden) excitations involved in charge separation. Experimental values (measured in $\mathrm{CHCl}_{3}$ ) are adapted from ref. [15]. Calculated data obtained at the PBE0/def2-SVP level of TDDFT.

\begin{tabular}{|c|c|c|c|c|c|c|c|}
\hline Compound & Excitation & Type & $f_{\text {osc }}$ & $E_{\text {calc }} / \mathrm{eV}$ & $E_{\text {exp }} / \mathrm{eV}$ & $\lambda_{\text {calc }} / \mathrm{nm}$ & $\lambda_{\text {exp }} / \mathrm{nm}$ \\
\hline \multirow[t]{14}{*}{ PPV-Ru1 } & $\mathrm{S}_{1}$ & PPV $\rightarrow$ Dye & 0.00 & 1.45 & - & 855 & - \\
\hline & $\mathrm{S}_{2}$ & PPV $\rightarrow$ Dye & 0.00 & 1.57 & - & 789 & - \\
\hline & $\mathrm{S}_{3}$ & PPV $\rightarrow$ Dye & 0.00 & 1.82 & - & 680 & - \\
\hline & $\mathrm{S}_{4}$ & PPV $\rightarrow$ Dye & 0.00 & 1.86 & - & 666 & - \\
\hline & $\mathrm{S}_{5}$ & PPV $\rightarrow$ Dye & 0.00 & 1.93 & - & 642 & - \\
\hline & $\mathrm{S}_{6}$ & PPV $\rightarrow$ Dye & 0.00 & 2.05 & - & 604 & - \\
\hline & $\mathrm{S}_{7}$ & PPV $\rightarrow$ Dye & 0.00 & 2.25 & - & 550 & - \\
\hline & $\mathrm{S}_{9}$ & PPV $\rightarrow$ Dye & 0.00 & 2.31 & - & 537 & - \\
\hline & $\mathrm{S}_{10}$ & PPV $\rightarrow$ Dye & 0.00 & 2.34 & - & 530 & - \\
\hline & $\mathrm{S}_{12}$ & PPV $\rightarrow$ Dye & 0.00 & 2.42 & - & 512 & - \\
\hline & $\mathrm{S}_{13}$ & $\pi \rightarrow \pi^{*}$ & 3.20 & 2.47 & 2.56 & 502 & 485 \\
\hline & $\mathrm{S}_{25}$ & $\mathrm{MLCT}$ & 0.17 & 2.81 & 2.60 & 441 & 476 \\
\hline & $\mathrm{S}_{35}$ & $\mathrm{MLCT}$ & 0.20 & 3.04 & 2.83 & 408 & 438 \\
\hline & $\mathrm{S}_{68}$ & $\pi \rightarrow \pi^{*}$ & 0.41 & 3.67 & 4.01 & 338 & 309 \\
\hline \multirow[t]{8}{*}{ PPV-Ru2 } & $\mathrm{S}_{1}$ & PPV $\rightarrow$ Dye & 0.00 & 1.62 & - & 766 & - \\
\hline & $\mathrm{S}_{4}$ & PPV $\rightarrow$ Dye & 0.00 & 2.16 & - & 573 & - \\
\hline & $\mathrm{S}_{5}$ & PPV $\rightarrow$ Dye & 0.00 & 2.21 & - & 562 & - \\
\hline & $\mathrm{S}_{6}$ & PPV $\rightarrow$ Dye & 0.00 & 2.31 & - & 537 & - \\
\hline & $\mathrm{S}_{8}$ & $\pi \rightarrow \pi^{*}$ & 3.27 & 2.48 & 2.58 & 500 & 481 \\
\hline & $\mathrm{S}_{16}$ & $\mathrm{MLCT}$ & 0.09 & 2.82 & 2.71 & 440 & 458 \\
\hline & $\mathrm{S}_{23}$ & $\mathrm{MLCT}$ & 0.12 & 3.01 & 2.88 & 412 & 430 \\
\hline & $\mathrm{S}_{51}$ & $\pi \rightarrow \pi^{*}$ & 0.63 & 3.68 & 3.79 & 337 & 327 \\
\hline
\end{tabular}

visualized by charge density difference (CDD) plots, see Figure 6.

Both experimental and calculated spectra are dominated by a peak at ca. $2.5 \mathrm{eV}$, which stems from a $\pi \rightarrow \pi^{*}$ transition localized on the PPV polymer. This excitation energy is in rather good agreement with the band gap of the extended polymer states derived from the periodic DFT calculations (see Figure 3). The intensity of this peak is possibly overestimated by the calculation due to the linear arrangement of the phenylenevinylene chain in the model system which amplifies the transition dipoles in the aromatic plane. The $\mathrm{Ru}(\mathrm{II})$ dye is hardly involved in this transition at all, as already identified in the analysis of the band structure, and it is also observed in the measured spectrum of pure PPV-related compounds. ${ }^{[15]}$

PPV-Ru1 as well as PPV-Ru2 feature a variety of moderately intense MLCT bands in the energetic region around $3 \mathrm{eV}-$ overlapping with the bright $\pi \rightarrow \pi^{*}$ transition in PPV. Therefore, a precise assignment of the predicted MLCT transitions with respect to the experimental data is hampered. Yet, these MLCT transitions are likely to be of fundamental importance for the charge separation process. Since these transitions are completely localized on the ruthenium photosensitizer, some insight can instead be gained from experimental measurements of the unfunctionalized Ru1 and Ru2 complexes. Compared to those values, calculated results are systematically blue-shifted by $0.1 \mathrm{eV}$ to $0.2 \mathrm{eV}$ which can likely be traced to the influence of the $\mathrm{CHCl}_{3}$ solvent used in the experiment. ${ }^{[15]}$

Furthermore, an additional $\pi \rightarrow \pi^{*}$ absorption feature is predicted around $4 \mathrm{eV}$ for both molecules, outside the visible spectrum. Therefore, and not surprisingly, the absorption spectra of PPV-Ru1 and PPV-Ru2 are governed by the separate photophysical properties of the polymer and the Ru-based photosensitizer, while any significant light-driven interactions between the polymer and the photosensitizer - i.e. strongly dipole-allowed CT states - are not observed. However, dipoleforbidden $\mathrm{CT}$ transitions would have negligible intensity in an experimental measurement and are optically inaccessible from the ground state and only experimentally detectable based on transient absorption techniques. Of particular interest is a series of dark charge transfer excitations present in both hybrid systems at slightly differing energies, starting from $1.45 \mathrm{eV}$ in PPV-Ru1 and from $1.62 \mathrm{eV}$ in PPV-Ru2 which are characterized by very low oscillator strengths. All of these states feature a distinct charge-separated nature, where an electron has been transferred from the PPV backbone to the ligand sphere of the respective ruthenium complex.

The TDDFT results provide a conclusive picture of possible charge separation processes. As all charge-separated states in the low energy region are dark, initial excitation must occur into a higher excited state such as via $\pi \rightarrow \pi^{*}$ (PPV-Ru1: $\mathrm{S}_{8}$; PPV-Ru2: $S_{13}$ ) or ${ }^{1} M L C T$ (PPV-Ru1: $S_{25}, S_{35} ;$ PPV-Ru2: $S_{16}, S_{23}$ ) excitation in the visible region between $2.5 \mathrm{eV}$ and $3 \mathrm{eV}$. From these states, the molecule can relax into a charge-separated state through internal conversion and/or intersystem crossing.

However, the pronounced excited state lifetimes of ${ }^{3} \mathrm{MLCT}$ states - rapidly accessible via intersystem crossing - suggests that the most important pathway to charge separation occurs through MLCT transitions. Experiments yield lifetimes of the charge-separated states of $(1587 \pm 201$ ps) for PPV-Ru1 and (994 \pm 203 ps) for PPV-Ru2. ${ }^{[15]}$ At these timescales, it is most likely that the charge-separated states have triplet character, which delays the spin-forbidden transition back towards the singlet ground state. 
Both PPV-Ru1 and PPV-Ru2 show certain similarities but also some characteristic differences in the TDDFT calculations. First, excitations centered on the aromatic polymer chain are hardly influenced by the presence of linker and photosensitizer, which is consistent with other findings both here (see, e.g., Figure 3) and elsewhere. ${ }^{[15]}$ Energies of the MLCT bands in the two compounds are also similar, in spite of the fact that oscillator strengths are higher for PPV-Ru1. The main differences lie in the low-lying dark states with charge transfer character. The $S_{1}$ state is lower in PPV-Ru1 by ca. $0.2 \mathrm{eV}$ than in PPV-Ru2, and similar findings hold for other states of this type. This trend is most easily attributed to the electron-withdrawing effect of the $-\mathrm{COOH}$ substituents on the photosensitizer. As a result, PPV-Ru1 features a higher number of low energy charge-separated states which are accessible upon internal conversion. Although the time scale of charge separation cannot be directly deduced from the density of states, we still speculate that this finding might also allow to explain why charge separation occurs faster in PPV-Ru1 (49 \pm 4 ps) than in PPV-Ru2 (86 \pm 9 ps). ${ }^{[15]}$

As highlighted above, the TDDFT predictions are in excellent agreement with both the results of the ground-state periodic DFT calculations and the experiment data. In particular, the ability to assess the overshadowed MLCT transitions as well as to elucidate dark charge-separated transitions is a valuable tool in predicting and analyzing the photophysics and subsequent excited state relaxation pathways in the present dyesensitized polymers.

\section{Conclusion}

Using both periodic density functional theory (DFT) calculations and local time-dependent DFT calculations, we have addressed the coupling of photoactive $\mathrm{Ru}(\mathrm{II})$-based complexes with two different substituents $\left(-\mathrm{CH}_{3}\right.$ vs. $\left.-\mathrm{COOH}\right)$ to a polymer matrix. Conducting polymers represent a promising alternative to semiconducting oxide electrodes typically used in dye-sensitized cathodes. Whereas the electronic properties of the Ru dye depend on the choice of the substituents, those of the polymer are insensitive to the presence of the attached dye. The electronic states of the polymer and the dye do not couple, hence the calculated photoinduduced electronic excitations, which agree well with experimental results, remain localized either on the polymer or the dye. However, we identify possible routes towards photoinduced excitations that would lead to a charge transfer from the polymer to the dye which might then for example be used to induce catalytic reactions at suitable metal centers attached to the dye.

\section{Acknowledgements}

Financial support by the German Science Foundation (DFG) through Project $\mathrm{C} 5$ of the Transregional Collaborative Research Centre TRR234 CataLight (Project No. 364549901) is gratefully acknowledged. Computer time on the JUSTUS cluster at UIm
University provided by the bwHPC initiative and the bwHPC-C5 project funded by the Baden-Württemberg government (MWK) and the German Research Foundation (DFG) through grant number INST 40/467-1 FUGG is gratefully acknowledged. Open Access funding enabled and organized by Projekt DEAL.

\section{Conflict of Interest}

The authors declare no conflict of interest.

Keywords: organic solar cells • photoactive complexes polymer matrix $\cdot$ quantum chemistry $\cdot$ electronic coupling

[1] R. Schlögl, ChemSusChem 2010, 3, 209-222.

[2] A. Blakers, N. Zin, K. R. McIntosh, K. Fong, Energy Procedia 2013, 33, 110, PV Asia Pacific Conference 2012.

[3] J. Zhao, A. Wang, M. A. Green, Prog. Photovoltaics 1999, 7, 471-474.

[4] J. Zhao, A. Wang, M. A. Green, Sol. Energy Mater. Sol. Cells 2001, 66, 2736 .

[5] B. O'Regan, M. Grätzel, Nature 1991, 353, 737-740.

[6] M. Liang, J. Chen, Chem. Soc. Rev. 2013, 42, 3453.

[7] A. Hagfeldt, G. Boschloo, L. Sun, L. Kloo, H. Pettersson, Chem. Rev. 2010, $110,6595-6663$.

[8] E. Maggio, G. C. Solomon, A. Troisi, ACS Nano 2013, 8, 409-418.

[9] W. H. Nguyen, C. D. Bailie, E. L. Unger, M. D. McGehee, J. Am. Chem. Soc. 2014, 136, 10996-11001.

[10] R. Stalder, D. Xie, A. Islam, L. Han, J. R. Reynolds, K. S. Schanze, ACS Appl. Mater. Interfaces 2014, 6, 8715-8722.

[11] A. Sen, A. Groß, Int. J. Quantum Chem. 2019, 119, e25963.

[12] A. Sen, A. Groß, ACS Appl. Energ. Mater. 2019, 2, 6341-6347.

[13] A. Sen, A. Groß, J. Phys. Chem. C 2020, 124, 8526-8540.

[14] A. B. Muñoz-García, M. Pavone, Phys. Chem. Chem. Phys. 2015, 17, $12238-12246$.

[15] R. A. Wahyuono, B. Seidler, S. Bold, A. Dellith, J. Dellith, J. Ahner, P. Wintergerst, G. Lowe, M. D. Hager, M. Wächtler, C. Streb, U. S. Schubert, S. Rau, B. Dietzek, Sci. Rep. 2021, 11, 2787.

[16] S. Kaufhold, L. Petermann, R. Staehle, S. Rau, Coord. Chem. Rev. 2015, 304-305, 73-87.

[17] K. E. Dalle, J. Warnan, J. J. Leung, B. Reuillard, I. S. Karmel, E. Reisner, Chem. Rev. 2019, 119, 2752-2875.

[18] M. G. Pfeffer, T. Kowacs, M. Wächtler, J. Guthmuller, B. Dietzek, J. G. Vos, S. Rau, Angew. Chem. Int. Ed. 2015, 54, 6627-6631; Angew. Chem. 2015, $127,6727-6731$.

[19] N. Hannewald, P. Winterwerber, S. Zechel, D. Y. W. Ng, M. D. Hager, T. Weil, U. S. Schubert, Angew. Chem. Int. Ed. 2021, 60, 6218-6229; Angew. Chem. 2021, 133, 6282-6294.

[20] I. Romanenko, A. Rajagopal, C. Neumann, A. Turchanin, C. Streb, F. H. Schacher, J. Mater. Chem. A 2020, 8, 6238-6244.

[21] S. P. Rittmeyer, A. Groß, Beilstein J. Nanotechnol. 2012, 3, 909-919.

[22] F. Zhang, M. Johansson, M. Andersson, J. Hummelen, O. Inganäs, Adv. Mater. 2002, 14, 662-665.

[23] M. Martynow, S. Kupfer, S. Rau, J. Guthmuller, Phys. Chem. Chem. Phys. 2019, 21, 9052-9060.

[24] V. Blum, R. Gehrke, F. Hanke, P. Havu, V. Havu, X. Ren, K. Reuter, M. Scheffl, Comp. Phys. Commun. 2009, 180, 2175-2196.

[25] V. Havu, V. Blum, P. Havu, M. Scheffler, J. Comput. Phys. 2009, 228, 8367-8379.

[26] X. Ren, P. Rinke, V. Blum, J. Wieferink, A. Tkatchenko, A. Sanfilippo, K. Reuter, M. Scheffler, New J. Phys. 2012, 14, 053020.

[27] A. Marek, V. Blum, R. Johanni, V. Havu, B. Lang, T. Auckenthaler, A. Heinecke, H.-J. Bungartz, H. Lederer, J. Condens. Matter Phys. 2014, 26, 213201.

[28] S. V. Levchenko, X. Ren, J. Wieferink, R. Johanni, P. Rinke, V. Blum, M. Scheffl, Comput. Phys. Commun. 2015, 192, 60-69.

[29] J. P. Perdew, K. Burke, M. Ernzerhof, Phys. Rev. Lett. 1996, 77, 3865-3868.

[30] A. Tkatchenko, M. Scheffler, Phys. Rev. Lett. 2009, 102, 073005.

[31] E. van Lenthe, E. J. Baerends, J. G. Snijders, J. Chem. Phys. 1994, 101, 9783-9792.

[32] C. Adamo, V. Barone, J. Chem. Phys. 1999, 110, 6158-6170. 
[33] C. Lee, W. Yang, R. G. Parr, Phys. Rev. B 1988, 37, 785-789.

[34] A. D. Becke, J. Chem. Phys. 1993, 98, 5648-5652.

[35] M. J. Frisch, G. W. Trucks, H. B. Schlegel, G. E. Scuseria, M. A. Robb, J. R. Cheeseman, G. Scalmani, V. Barone, G. A. Petersson, H. Nakatsuji, X. Li, M. Caricato, A. V. Marenich, J. Bloino, B. G. Janesko, R. Gomperts, B. Mennucci, H. P. Hratchian, J. V. Ortiz, A. F. Izmaylov, J. L. Sonnenberg, D. Williams-Young, F. Ding, F. Lipparini, F. Egidi, J. Goings, B. Peng, A. Petrone, T. Henderson, D. Ranasinghe, V. G. Zakrzewski, J. Gao, N. Rega, G. Zheng, W. Liang, M. Hada, M. Ehara, K. Toyota, R. Fukuda, J. Hasegawa, M. Ishida, T. Nakajima, Y. Honda, O. Kitao, H. Nakai, T. Vreven, K. Throssell, J. A. Montgomery, Jr., J. E. Peralta, F. Ogliaro, M. J. Bearpark, J. J. Heyd, E. N. Brothers, K. N. Kudin, V. N. Staroverov, T. A. Keith, R Kobayashi, J. Normand, K. Raghavachari, A. P. Rendell, J. C. Bu- rant, S. S lyengar, J. Tomasi, M. Cossi, J. M. Millam, M. Klene, C. Adamo, R. Cammi, J. W. Ochterski, R. L. Martin, K. Morokuma, O. Farkas, J. B. Foresman, D. J. Fox, Gaussian $\sim 16$ Revision C.01, 2016, Gaussian Inc. Wallingford CT.

[36] S. Grimme, S. Ehrlich, L. Goerigk, J. Comb. Chem. 2011, 32, 1456-1465.

[37] A. V. Marenich, C. J. Cramer, D. G. Truhlar, J. Phys. Chem. B 2009, 113 6378-6396.

[38] J. Preat, A. Hagfeldt, E. A. Perpéte, Energy Environ. Sci. 2011, 4, 45374549.

[39] C. Li, H. Wonneberger, Adv. Mater. 2012, 24, 613-636.

[40] J. Wang, S. Cong, S. Wen, L. Yan, Z. Su, J. Phys. Chem. C 2013, 117, 22452251.

[41] T. Yanai, D. P. Tew, N. C. Handy, Chem. Phys. Lett. 2004, 393, 51-57.

[42] D. J. Tozer, R. D. Amos, N. C. Handy, B. O. Roos, L. Serrano-Andres, Mol. Phys. 1999, 97, 859-868.

[43] A. Dreuw, J. L. Weisman, M. Head-Gordon, J. Chem. Phys. 2003, 119, 2943-2946.

[44] S. Kupfer, J. Guthmuller, M. Wächtler, S. Losse, S. Rau, B. Dietzek, J. Popp, L. González, Phys. Chem. Chem. Phys. 2011, 13, 15580-15588.

[45] C. Müller, M. Schulz, M. Obst, L. Zedler, S. Gräfe, S. Kupfer, B. Dietzek, J. Phys. Chem. A 2020, 124, 6607-6616.

[46] F. Weigend, R. Ahlrichs, Phys. Chem. Chem. Phys. 2005, 7, 3297-3305.

[47] F. Neese, Wiley Interdiscip. Rev.: Comput. Mol. Sci. 2012, 2, 73-78.

[48] F. Neese, Wiley Interdiscip. Rev.: Comput. Mol. Sci. 2018, 8, e1327.

[49] E. Aprà, E. J. Bylaska, W. A. de Jong, N. Govind, K. Kowalski, T. P. Straatsma, M. Valiev, H. J. J. van Dam, Y. Alexeev, J. Anchell, V. Anisimov, F. W. Aquino, R. Atta-Fynn, J. Autschbach, N. P. Bauman, J. C. Becca, D. E. Bernholdt, K. Bhaskaran-Nair, S. Bogatko, P. Borowski, J. Boschen, J. Brabec, A. Bruner, E. Cauët, Y. Chen, G. N. Chuev, C. J. Cramer, J. Daily M. J. O. Deegan, T. H. Dunning, M. Dupuis, K. G. Dyall, G. I. Fann, S. A Fischer, A. Fonari, H. Früchtl, L. Gagliardi, J. Garza, N. Gawande, S. Ghosh, K. Glaesemann, A. W. Götz, J. Hammond, V. Helms, E. D. Hermes, K. Hirao, S. Hirata, M. Jacquelin, L. Jensen, G. Johnson, H. Jónsson, R. A Kendall, M. Klemm, R. Kobayashi, V. Konkov, S. Krishnamoorthy, M. Krishnan, Z. Lin, R. D. Lins, R. J. Littlefield, A. J. Logsdail, K. Lopata, W.
Ma, A. V. Marenich, J. Martin del Campo, D. Mejia-Rodriguez, J. E. Moore, J. M. Mullin, T. Nakajima, D. R. Nascimento, J. A. Nichols, P. J. Nichols, J. Nieplocha, A. O. de-la Roza, B. Palmer, A. Panyala, T. Pirojsirikul, B. Peng, R. Peverati, J. Pittner, L. Pollack, R. M. Richard, P. Sadayappan, G. C Schatz, W. A. Shelton, D. W. Silverstein, D. M. A. Smith, T. A. Soares, D. Song, M. Swart, H. L. Taylor, G. S. Thomas, V. Tipparaju, D. G. Truhlar, K. Tsemekhman, T. Van Voorhis, Á. Vázquez-Mayagoitia, P. Verma, O. Villa, A. Vishnu, K. D. Vogiatzis, D. Wang, J. H. Weare, M. J. Williamson, T. L. Windus, K. Woliński, A. T. Wong, Q. Wu, C. Yang, Q. Yu, M. Zacharias, Z. Zhang, Y. Zhao, R. J. Harrison, J. Chem. Phys. 2020, 152, 184102.

[50] D. Andrae, U. Häußermann, M. Dolg, H. Stoll, H. Preuß, Theor. Chim. Acta 1990, 123-141.

[51] S. Hirata, M. Head-Gordon, Chem. Phys. Lett. 1999, 314, 291-299.

[52] H. Dhifaoui, M. Pegu, W. Aloui, A. Bouazizi, S. Kazim, S. Ahmad, Emerg. Mater. Res. 2020, 3, 687-692.

[53] L. Feng, F. Wang, M.-S. Niu, F. Zheng, P.-Q. Bi, X.-Y. Yang, W.-L. Xu, X.-T. Hao, Opt. Mater. Express 2017, 7, 687.

[54] C.-S. Wu, C.-T. Liu, Y. Chen, Polym. Chem. 2012, 3, 3308.

[55] Y. Sohn, Vacuum 2014, 99, 107-109.

[56] D. Mahlberg, S. Sakong, K. Forster-Tonigold, A. Groß, J. Chem. Theory Comput. 2019, 15, 3250-3259.

[57] J. P. Perdew, A. Zunger, Phys. Rev. B 1981, 23, 5048-5079.

[58] J. M. Crowley, J. Tahir-Kheli, W. A. Goddard, J. Phys. Chem. Lett. 2016, 7, 1198-1203.

[59] S. Fantacci, F. De Angelis, A. Selloni, J. Am. Chem. Soc. 2003, 125, 43814387.

[60] M.-F. Charlot, A. Aukauloo, J. Phys. Chem. A 2007, 111, 11661-11672.

[61] G. Kudur Jayaprakash, B. E. Kumara Swamy, S. Sharma, J. J. SantoyoFlores, Microchem. J. 2020, 158, 105116.

[62] J. Sworakowski, Synth. Met. 2018, 235, 125-130.

[63] A. A. Adeniyi, T. L. Ngake, J. Conradie, Electroanalysis 2020, 32, 26592668.

[64] F. F. Salomón, N. C. Vega, T. Parella, F. E. M. Vieyra, C. D. Borsarelli, C. Longo, M. Cattaneo, N. E. Katz, ACS Omega 2020, 5, 8097-8107.

[65] R. Patra, A. Maity, K. K. Rajak, J. Chem. Sci. 2020, 132, 140.

[66] J. J. Sutton, D. Preston, P. Traber, J. Steinmetzer, X. Wu, S. Kayal, X.-Z. Sun, J. D. Crowley, M. W. George, S. Kupfer, K. C. Gordon, J. Am. Chem. Soc. 2021, 143, 9082-9093.

[67] S. Kupfer, D. Kinzel, M. Siegmann, J. Philipp, B. Dietzek, S. Gräfe, J. Phys. Chem. C 2018, 122, 3273-3285.

Manuscript received: July 30, 2021

Accepted manuscript online: November 11, 2021

Version of record online: November 24, 2021 